\title{
INITIAL TIDAL EFFECTS ON SHELL FORMATION
}

\author{
R. A. James and Althea Wilkinson, \\ Department of Astronomy, \\ University of Manchester, Manchester, M13 9PL, England.
}

\section{INTRODUCTION}

Sharp edged features have been observed by Malin and Carter (1980), Schweizer (1980) and others in the envelopes of many elliptical galaxies. The most promising of current models involves the disruption of a low mass galaxy penetrating close to the centre of a considerably larger ellipsoidal galaxy (Schweizer, 1980, Quinn, 1984, Hernquist and Quinn, 1986, Dupraz and Combes, 1986). The release of stars having a range of energies and with a definite relationship between velocity and position at the point of release generates sharp features by the "phase wrapping" mechanism.

We examine this effect using a self-consistent particle-mesh code to follow the disruption phase in an almost radial encounter. A standard 2-body code continues the integration up to a Hubble time.

\section{NUMERICAL TECHNIQUES}

The initial self-consistent model was constructed by the procedure of Wilkinson and James (1982) which allows us to specify the initial ratio of kinetic energy $T$ to potential energy $V$. Low values of this ratio lead to compact structures following the de Vaucouleurs $r^{1 / 4}$ law as far as mesh resolution permits (Grimshaw, 1985). The preliminary calculations reported here use 25000 particles and a potential mesh of dimension $33 \times 33 \times 33$ points with a linear scale of $1 \mathrm{kpc}$ per mesh interval and a time step of $2 \times 10^{6}$ years.

We assume that the primary is a Plummer sphere with scale radius $5 \mathrm{kpc}$ and mass $10^{44}$ grams. The secondary galaxies all start with mass centres at distance 100 $\mathrm{kpc}$ from the centre of the primary and with initial systematic velocities directed towards this centre.

\section{RESULTS}

A pair of parabolic encounters with a secondary of mass $10^{42}$ grams shows the effect of the central concentration of the secondary on the break-up. The disruption is a relatively smooth process for an extended object with an initial energy ratio $2 T / V$ of 0.5 and a half-mass radius of $4.5 \mathrm{kpc}$. It is essentially complete when the secondary enters the $5 \mathrm{kpc}$ sphere defining the primary core. For a compact object with initial energy ratio 0.1 and half-mass radius $1.9 \mathrm{kpc}$, there is a well- 
marked disruption phase centred on the transit of the secondary through the centre of the primary. About 60 percent of the secondary is stripped before this stage, and a further 20 percent is released in $2.6 \times 10^{6}$ years. In contrast, a more inassive compact model $\left(5 \times 10^{42}\right.$ grams) loses only 12 percent of its mass in $1.6 \times 10^{6}$ years, emerging with 46 percent of its initial mass still bound. The transition to the 2body calculation assumes that all stars are unbound, thus introducing a problem in separating stars released during disruption from those which still belong to the secondary. The continuations rum to date pick up the model soon after transit and those for the massive secondary are dominated by bound rather than unbound stars. Future code modifications will enable us to separate out the stars released near transit, but at present we restrict ourselves to low mass secondarics. Most of our models generate some shell structure after the disruption phase. The general trend is that shell structure is richer for more compact secondaries and for those with more elliptic orbits. The richest structure is observed for the most elliptic of our orbits with a compact secondary. This has an apocentre distance of 158 kpc and shows 7 visible shells $7.4 \times 10^{9}$ years after disruption.

\section{CONCLUSIONS}

The phase wrapping mechanism remains a plausible explanation of shell structure around elliptical galaxies. The efficiency of shell formation is greater with bound companions than in the parabolic case. Compact secondaries give stronger shells than the more extended ones and this is correlated with differences in their disruption history. The disruption process depends on the relative primary and secondary length scales and further work is required to establish if secondary structure is the dominant influence.

The main limitations of our work are the limited resolution employed so far and our inability to deal with more massive and less completely disrupted secondaries correctly. Both these limitations will be removed in future.

\section{ACKNOWLEDGEMENTS}

It is a pleasure to thank Lars Hernquist and P. J. Quinn and also Ch. Dupraz and $F$. Combes for communicating their results to us in advance of publication.

\section{REFERENCES}

Dupraz, Ch. \& Combes, F., 1986, preprint.

Grimshaw, L., 1985, Ph.D. Thesis, University of Manchester.

Hernquist, L. \& Quinn, P. J., 1986, preprint.

Malin, D.F., \& Carter, D., 1980, Nature,285, 643.

Quinn, P. J., 1984, Astrophys. J., 279, 596.

Schweizer, F., 1980, Astrophys. J., 237, 303.

Wilkinson, A. \& James, R. A., 1982, M.N.R.a.S., 199, 171. 